\title{
MicroRNA dysregulation as a prognostic biomarker in colorectal cancer
}

\author{
This article was published in the following Dove Press journal: \\ Cancer Management and Research \\ 14 October 2014 \\ Number of times this article has been viewed
}

\author{
Yujuan Dong ${ }^{1,2}$ \\ Jun $\mathrm{Yu}^{2}$ \\ Simon SM $\mathrm{Ng}^{1,2}$ \\ 'Division of Colorectal Surgery, \\ Department of Surgery, Prince \\ of Wales Hospital, The Chinese \\ University of Hong Kong, Hong Kong; \\ ${ }^{2}$ Institute of Digestive Disease, State \\ Key Laboratory of Digestive Disease, \\ Li Ka Shing Institute of Health \\ Sciences, The Chinese University \\ of Hong Kong, Hong Kong
}

\begin{abstract}
Colorectal cancer (CRC) is one of the most potentially curable cancers, yet it remains the fourth most common overall cause of cancer death worldwide. The identification of robust molecular prognostic biomarkers can refine the conventional tumor-node-metastasis staging system, avoid understaging of tumor, and help pinpoint patients with early-stage CRC who may benefit from aggressive treatments. Recently, epigenetic studies have provided new molecular evidence to better categorize the CRC subtypes and predict clinical outcomes. In this review, we summarize recent findings concerning the prognostic potential of microRNAs (miRNAs) in CRC. We first discuss the prognostic value of three tissue miRNAs (miR-21-5p, miR-29-3p, miR-148-3p) that have been examined in multiple studies. We also summarize the dysregulation of miRNA processing machinery DICER in CRC and its association with risk for mortality. We also reviewe the potential application of miRNA-associated single-nucleotide polymorphisms as prognostic biomarkers for CRC, especially the miRNA-associated polymorphism in the KRAS gene. Last but not least, we discuss the microsatellite instability-related miRNA candidates. Among all these candidates, miR-21-5p is the most promising prognostic marker, yet further prospective validation studies are required before it can go into clinical usage.
\end{abstract}

Keywords: microRNA, colorectal cancer, prognostic biomarker, single-nucleotide polymorphism, microsatellite instability

\section{Introduction}

Colorectal cancer (CRC) is a malignant neoplasm affecting the lower gastrointestinal tract. CRC includes two major entities: colon cancer (CC), the malignance in the inner wall of the colon that constitutes two-thirds to three-quarters of all CRC cases; and rectal cancer (RC), defined as cancer located within $12 \mathrm{~cm}$ or less from the anal verge. CRC is a global public health problem: it is the third most common cancer and the fourth leading cause of cancer-related deaths in the world, with an estimated incidence of 1.2 million new cases and a mortality of $>600,000$ deaths annually $(8 \%$ of all cancer deaths). ${ }^{1}$ Both the incidence and death rates from $\mathrm{CRC}$ are increasing rapidly in Asian countries. ${ }^{2}$

Currently, the clinicopathologic tumor staging based on the tumor-node-metastasis (TNM) system is the basic prognostic marker for CRC clinical outcomes. The TNM system describes the degree to which the tumor has invaded the bowel wall and spread to the regional lymph nodes as well as to distant organs. ${ }^{3}$ Although the TNM staging system is the mainstay of prognostication, this classification has weaknesses. Inadequate examination of lymph nodes may lead to understaging of the tumor and subsequent treatment failure. ${ }^{3}$ Moreover, histologically identical CRC patients may have different 
genetic and epigenetic backgrounds that lead to distinctive disease progression and clinical outcomes. For example, TNM stage II patients with no lymph node metastasis have relatively better outcomes. However, approximately onefourth of these patients can still have high risk for disease relapse after surgical resection. Unfortunately, no prognostic marker is currently available for identifying the patients who should benefit from more-aggressive treatments. ${ }^{4}$ Recent epigenetic studies suggested that microRNAs (miRNAs) may help to better categorize the CRC subtypes and predict the outcomes.

miRNAs belong to a class of highly conserved $\sim 22-$ nucleotide single-stranded RNAs that epigenetically regulate protein translation through binding to the $3^{\prime}$ untranslated region (UTR) of target messenger RNA (mRNA) and mediate either mRNA degradation or translational repression. ${ }^{5}$ A single miRNA can manipulate multiple target gene expressions, initiate signaling pathways, and provoke signal crosstalk. It is estimated that miRNAs can fine-tune up to one-third of human gene translations. ${ }^{6}$ By targeting multiple transcripts, miRNAs can epigenetically regulate fundamental cellular processes, such as cell proliferation, apoptosis, differentiation, and migration, which strongly indicates that they may function as potential oncogenes or tumor suppressors in cancer development. Indeed, a global impairment of miRNA has been described in various human cancers, including $\mathrm{CRC}$. $^{7,8} \mathrm{~A}$ spectrum of dysregulated miRNAs was identified to be associated with CRC genesis, progression, and therapeutic response.

Herein, we summarize recent findings and discuss the potential value of miRNAs as prognostic biomarkers for CRC. For miRNA as a diagnostic marker and its therapeutic potential, readers can refer to recent reviews written by our group and others. ${ }^{9-11}$ It is worth pointing out that in 2011 miRBase adopted a new " $-5 \mathrm{p} /-3 \mathrm{p}$ ” miRNA nomenclature to replace the conventional $\mathrm{miR} / \mathrm{miR}^{*}$ notation (http://www. mirbase.org). In this review, we will use the most updated miRNA identification nomenclature and list the original name used in the literature as reference.

\section{miRNAs as prognostic biomarkers for CRC miR-2I-5p}

miR-21-5p (accession number: MIMAT0000076), previously named miR-21, is one of the most abundantly expressed oncogenic miRNAs in CRC,,$^{12,13}$ and has been extensively investigated for its prognostic potential in at least ten independent trials involving 2,039 patients since 2008 (Table 1). ${ }^{14-23}$
Slaby et $\mathrm{al}^{24}$ first reported that elevated levels of miR-21-5p significantly correlated with lymph node positivity and the development of distance metastasis in a small cohort of 29 CRC patients, suggesting the potential prognostic value of miR-21-5p in CRC. This hypothesis was further tested by Schetter et $\mathrm{al}^{14}$ in their multicenter study. Utilizing miRNA array profiling of 84 tumors and paired adjacent normal tissues, they identified 37 abnormal miRNAs, of which five promising miRNAs (miR-20a-5p [miR-20a], miR-21-5p, miR-106a-5p [miR-106a], miR-181b-5p [miR-181b], and miR-203a [miR-203]) were associated with unfavorable outcomes in the test cohort. Further quantitative real-time polymerase chain reaction (qRT-PCR)-based validation suggested that high miR-21-5p expression in tumor was significantly associated with a worse 5-year cancer-specific survival rate independent of demographic and clinicopathologic factors in a test cohort of 71 patients with sporadic colon adenocarcinomas. Moreover, the association of high miR-21-5p expression level in tumor and poor prognosis was confirmed by an external cohort of 103 colon adenocarcinoma patients from Hong Kong. ${ }^{14}$

The consistency of these associations has been proven by subsequent studies. Nielsen et a ${ }^{15}$ performed a retrospective study based on a multicenter Danish and Scottish randomized clinical trial (RANX05) involving 130 stage II CC patients and 67 stage II RC patients. They evaluated the miR-21-5p expression using in situ hybridization on formalin-fixed paraffin-embedded tissue samples followed by image semiquantitative analysis. Strong staining of miR-21-5p was significantly associated with shorter disease-free survival (DFS) and overall survival (OS) in stage II CC patients. By multivariate analysis, the intense signal of miR-21-5p was a prognostic factor for stage II CC group after adjustment for other clinical parameters, including tumor histology, $K R A S$ mutational status, and microsatellite instability (MSI) status. Shibuya et a ${ }^{16}$ further validated the prognostic role of miR-21-5p in a cohort of 156 CRC patients in Japan. They concluded that a high level of miR-21-5p was associated with venous invasion, liver metastasis, advanced Dukes' stage, and a marginal link with lymph-node metastasis using the mean expression as a cutoff value. The group with higher levels of miR-21-5p had significantly shorter 5-year DFS and worse OS after multivariate regression. However, the authors did not specify the percentage of rectal cancer cases in their study cohort.

Besides serving as a single marker, miR-21-5p has been combined with other potential indicators to improve prognostic accuracy. One year after their first report about 


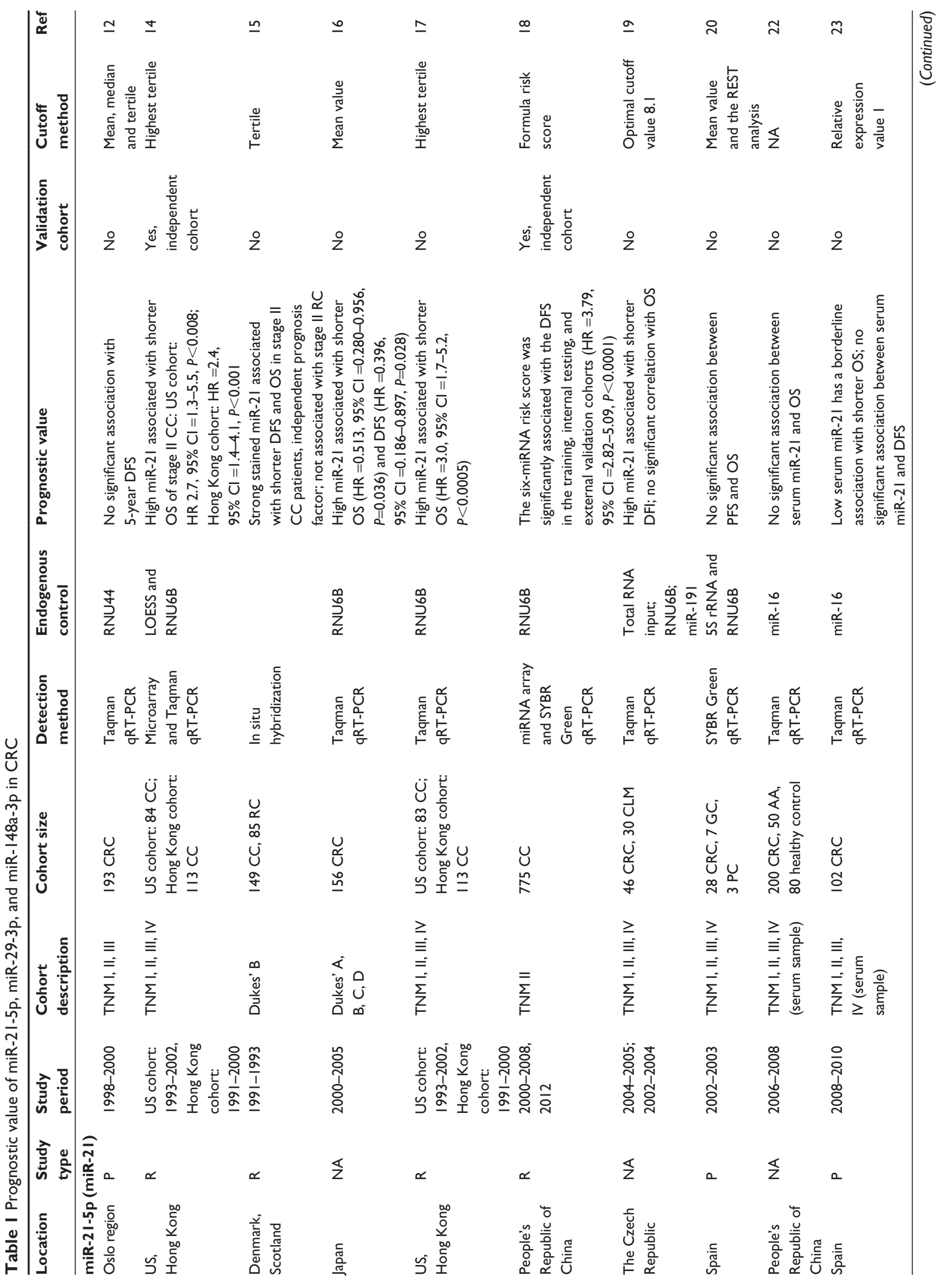




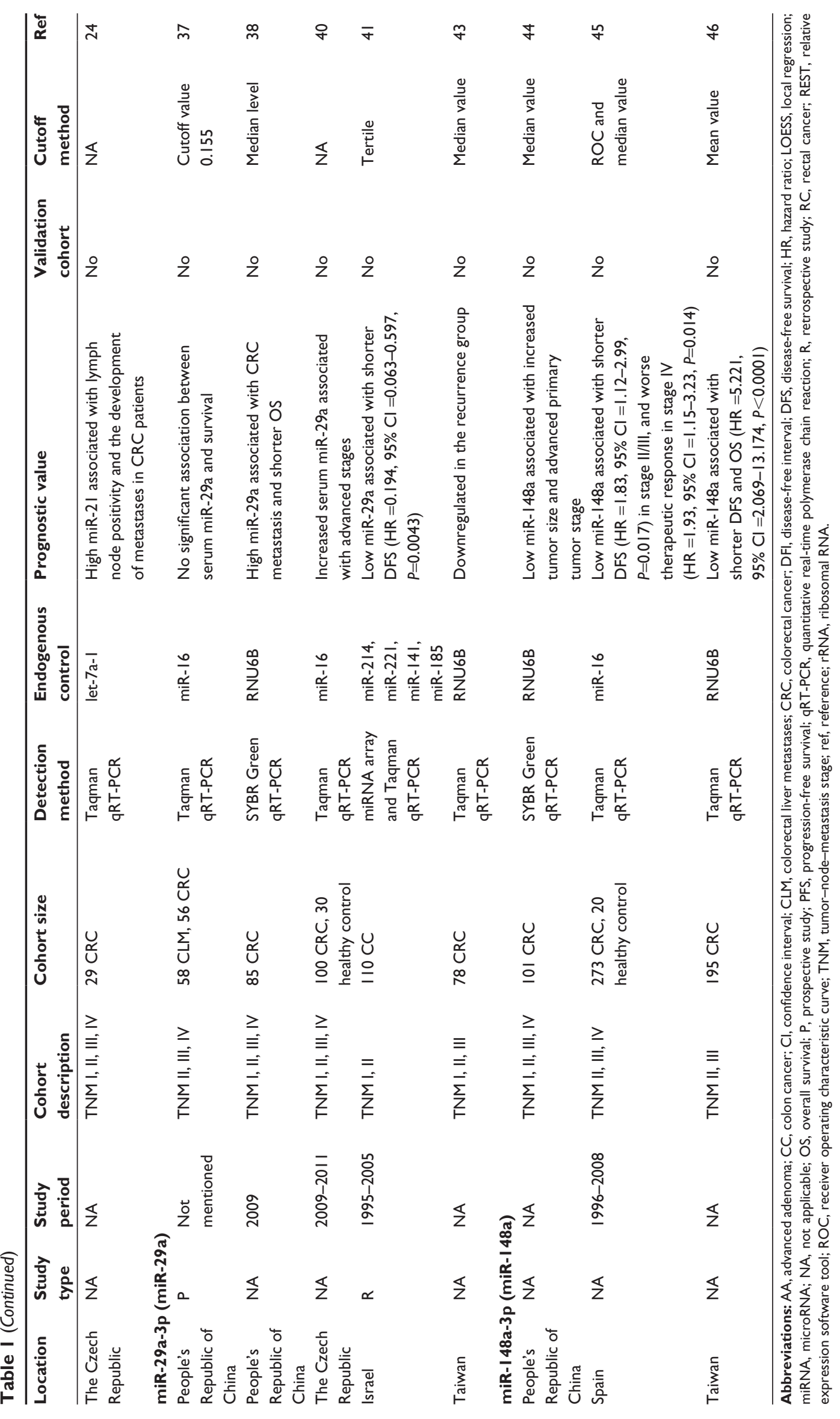


the prognostic values of miR-21-5p, Schetter et $\mathrm{al}^{17}$ conducted a consecutive study to detect its prognostic value in combination with a panel of nine inflammatory-related genes (PRG1, IL10, CD68, IL23a,IL12a, ANXA1, IL17a, $F O X P 3$, and $H L A-D R A)$ utilizing the same cohorts as described earlier. ${ }^{14}$ Consistent with their previous report, ${ }^{14}$ miR-21-5p retained its strong association with stage II CC cancer-specific mortality with the updated 1-year followup. They observed that the combination of high miR-21-5p level and high inflammatory risk score (IRS) could predict the unfavorable outcomes of either all stages or the subset of stage II CC. Despite the fact that miR-21-5p expression was associated with two inflammatory-related genes (IL10 and $I L 12 a$ ) in the IRS model, both miR-21-5p and IRS were found to be independent prognostic factors (adjusted for TNM stage) on multivariate analysis.

Most recently, Zhang et $\mathrm{al}^{18}$ carried out the largest multicenter retrospective trial to date to dissect the association between miRNA and stage II CC outcomes in a Chinese population. In their study, miRNA array identified $35 \mathrm{miR}-$ NAs as highly dysregulated in stage II CC. They further selected six potential indicator miRNAs (four upregulated miRNAs in cancer: miR-21-5p, miR-20a-5p, miR-103a-3p, and miR-106b-5p, and two downregulated miRNAs in cancer: miR-143-5p and miR-215) using the least absolute shrinkage and selection operator Cox regression model. ${ }^{18,25}$ They then developed a formula to calculate the disease recurrence risk score based on the expression levels of the six miRNAs and dichotomized patients into high-risk and low-risk groups. The high-risk panel score was significantly associated with poor prognosis: among an internal testing group of 137 stage II patients, $43 \%$ of the high-risk patients developed recurrence after a 5-year follow-up, whereas recurrence only occurred in $15 \%$ of the low-risk patients. Similarly, among an external validation set of 460 patients, $46 \%$ of the high-risk patients experienced relapse, whereas only $15 \%$ of the low-risk group had progressive disease. The six-miRNA panel as a predictor for 5-year DFS was independent of conventional clinicopathologic risk factors. They suggested that the combination of miR-21-5p with other indicators significantly enhanced the prognostic accuracy for $\mathrm{CC}$.

However, among the top aberrant miRNAs identified by the two large-scale miRNA screening studies mentioned above, ${ }^{14,18}$ only nine (miR-17-5p, miR-20a-5p, miR-21-5p, miR-92a-3p, miR-106b-5p, miR-181b-5p, miR-203a, miR-215, and miR-221-3p) in Zhang et al's China cohort overlapped with Schetter et al's US cohort. ${ }^{14,18}$ Specifically, the two miRNAs (miR-103a-3p and miR-143-5p) in Zhang et al's risk score panel were not considered to be dramatically altered in the US cohort. Although technical variations, such as different miRNA array platforms used and bioinformatics methods applied for data mining, may partially explain the inconsistency, it is possible that miRNA prognostic signature may differ across populations. Previous studies also suggested that miRNA transcriptome varied according to tumor sites and molecular alterations, such as CpG island methylator phenotype, MSI, KRAS, and TP53 status. ${ }^{26,27}$ Considering that their findings were restricted only to the Chinese population and specific CC subtypes, the generalizability of the multimarker signature on other ethnicities and subgroups still needs further validation.

miR-21-5p was significantly overexpressed in colon adenomas and adenocarcinoma. ${ }^{14}$ Initially it was identified to be upregulated in colonic epithelial cells. ${ }^{14}$ Further studies indicated that miR-21-5p was predominantly overexpressed in cancer-associated fibroblasts in CRC. ${ }^{15,28}$ Laboratory evidence of its role in CRC progression through fibroblastto-myofibroblast transdifferentiation provided coherence to the abovementioned epidemiological findings. ${ }^{29,30}$ Although multiple studies supported miR-21-5p as a promising CC prognostic marker, it is uncertain whether miR-21-5p is of relevance in certain clinical stages. Studies of its role in other less-common histologic subtypes, such as signet ring cell carcinoma, are also scant. Moreover, the association between miR-21-5p and RC is conflicting. Despite comparable expression levels and patterns in $\mathrm{CC}$ and $\mathrm{RC}$, miR-21-5p failed to predict the outcomes of patients with stage II RC in Nielson et al's study. ${ }^{15}$ No or reverse correlation of miR-21-5p with disease progression and mortality were also observed in several studies with heterogeneous population covering both CC and RC..$^{12,13,20-23}$ The contradictory findings might be due to inadequate sample size, insufficient follow-up time, and different medical intervention for the $\mathrm{CC} / \mathrm{RC}$ patients, but may also be rooted in the different molecular pathways for $\mathrm{CC} / \mathrm{RC}$ metastasis.

\section{miR-29a-3p}

The prognostic value of miR-29a-3p (previous name: miR-29a; accession number: MIMAT0000086) in CRC is not straightforward. Several studies suggested that miR29a-3p was significantly elevated in primary CRC compared with the matched adjacent normal tissue. ${ }^{31-34}$ Higher levels of plasma miR-29a-3p were also detected in CRC and advanced adenoma patients compared with normal healthy donors. ${ }^{35,36}$ Liver is one of the most common sites for CRC distant metastatic spread. Further study indicated that both 
serum and tissue miR-29a-3p were significantly higher in colorectal liver metastatic patients than in nonmetastatic patients, suggesting that miR-29a-3p might be associated with disease progression. ${ }^{37}$ In line with the prior study, ${ }^{37}$ another China-based single-centered study observed a higher miR-29a-3p level in the primary tumor tissues of M1-stage patients compared to those of M0-stage patients. ${ }^{38}$ They found that miR-29a-3p high expression correlated with CRC metastasis and poor OS. They also reported experimental evidence that overexpression of miR-29a-3p regulated Kruppel-like factor 4/matrix metalloproteinase-2/cadherin 1 cascade, and promoted cell invasion and dissemination in vitro and in vivo. ${ }^{38}$ Although it has not been published in a peer reviewed journal, a United States patent (US8338106 B2) claimed that the tumor:normal ratio of miR-29a-3p was shown to be an independent predictive marker of $\mathrm{CC}$ prognosis. ${ }^{39}$ A higher tumor:normal ratio of miR-29a-3p was associated with significantly worse DFS in a cohort of 77 CC patients. ${ }^{39}$

Meanwhile, several independent studies suggested a completely opposite prognostic value of miR-29a-3p. Although gradual increase of serum miR-29a-3p expression was associated with advanced stages of CRC, Faltejskova et $\mathrm{al}^{40}$ documented a comparable expression of miR-29a-3p in primary CRC serum and healthy subject serum in a Caucasian population. Weissmann-Brenner et $\mathrm{al}^{41}$ performed a retrospective study in a cohort of 110 early-stage CC patients who had not received adjuvant systemic therapy. They classified those patients who developed locoregional or distant recurrence within 36 months after initial complete resection into the poor-prognosis groups. On the basis of miRNA screening and a 10-year follow-up, they identified a significantly lower level of miR-29a-3p in stage II patients with poor prognosis. Decreased miR-29a in tumor was strongly associated with shorter DFS for stage II patients, which was independent of tumor grade and location. ${ }^{41}$ Despite the high specificity and sensitivity for miR-29a-3p in discrimination of good and poor prognosis for stage II CC, miR-29a-3p was incapable of predicting the clinical outcome of stage I CC patients.

Lee et $\mathrm{al}^{42}$ developed a reverse engineering approach (IMRE) to predict the altered expression of microRNAs using the currently available genome-wide gene expression datasets. This IMRE algorithm is based on the in-silicon miRNA target prediction databases, and the assumption that all miRNAs generally induce target cleavage and therefore inversely correlate with target mRNA level. Using four published human CRC gene expression array datasets (GSE12032, GSE17538, GSE4526, and GSE17181), Kuo et $\mathrm{al}^{43}$ performed a pooled IMRE computational analysis to infer putative recurrence-related miRNAs. IMRE identified miR-29a-3p and miR-29c-3p (miR-29c) as potential recurrence candidate markers for both stage II/III CRC patients. To verify their in-silicon prediction, they experimentally tested the miR-29a-3p/29c-3p expression level in $43 \mathrm{CRC}$ patients who experienced early recurrence within 1 year after curative surgery and 35 patients who remained free of disease progression. Kaplan-Meier analysis suggested that lower level of miR-29a-3p was significantly associated with early recurrence. ${ }^{43}$ However, no multivariate analysis was performed in this study. Whether miR-29a-3p is an independent prognosis factor needs further investigation.

Due to the insufficient evidence from both sides, current studies have not yet yielded a clear-cut picture of the miR-29a-3p dysregulation and its prognostic value in CRC. There are several possible explanations for this observation. First, these contradictory findings could be explained by the less-informative clinical data, especially lack of the definition of "healthy" control subjects. Some studies ${ }^{37,38}$ did not specify whether the patients enrolled accepted any radiotherapy or chemotherapy prior to specimen collection, which will very likely affect the miRNA expression. The clinical endpoints among studies ${ }^{41,43}$ varied as well. Second, the miR-29a-3p expression pattern in colorectal tissue is largely unknown. Considering the different percentages of stromal tissues in normal tissue and its cancerous counterpart, a simple qRTPCR based on RNA isolated from whole surgical specimens may distort the result. Therefore, an in situ hybridization or a qRT-PCR analysis with laser-captured stromal or epithelial compartment is necessary for carefully determining the source and expression pattern of miR-29a-3p. Third, several studies also suffered from flaws like heterogeneous populations and failure to stratify the $\mathrm{CC}$ and $\mathrm{RC}$, the two distinct clinical entities. An miRNA array study based on $57 \mathrm{RC}$ cases suggested that miR-29a-3p showed no significant difference between RC tissues and adjacent normal mucosa. ${ }^{26}$ Lack of stratification may have led to those contradictory findings. Fourth, most studies mentioned above $\mathrm{e}^{37,38,43}$ were based on very small sample sizes, as shown in Table 1. None of the studies gave any justification for the sample size used, which may bring type I and II errors in analysis. Last but not least, population ethnicity may be one of the potential confounding variables. Future strictly designed studies are certainly warranted.

\section{miR-I 48a-3p}

miR-148a-3p (previous name: miR-148a; accession number: MIMAT0000243) showed reduced expression in gastrointestinal 
cancer. ${ }^{44}$ Further study suggested that miR-148a-3p presented a comparable level between normal colonic mucosa and CRC tissues in stage II disease, whereas significant downregulation of miR-148a-3p was observed in more-advanced stages of CRC. ${ }^{45}$ This suggests that dysregulation of miR-148a-3p is one of the later events in CRC progression. Although tissue miR-148a-3p levels were not associated with 5-year DFS or OS in the stage II group, lower miR-148a-3p expression was significantly associated with worse 5-year DFS in stage III CRC. The group with low miR-148a-3p expression showed a trend toward a worse progression-free survival (PFS) and significantly worse OS in stage IV patients. After a statistical correction for multivariate testing, miR-148a-3p expression status was still independently associated with unfavorable outcomes for stage III/IV patients. ${ }^{45}$ Tsai et al ${ }^{46}$ tested the miR-148a-3p expression level in a Chinese population and observed a 2.5 -fold decrease in the expression in the earlyrelapse patients than in the late-relapse patients. Similar to the prior study, ${ }^{45}$ they observed strong associations between a lower miR-148a-3p level and worse DFS and OS in a cohort of 110 stage II/III patients. They also reported experimental evidence that overexpression of miR-148a-3p inhibited cell migration but not invasion. These available findings suggest that miR-148a-3p expression status has potential as a prognostic biomarker for advanced-stage CRC. Further replication studies are needed for validation.

Great efforts have been taken to identify new prognostic miRNA biomarkers for CRC. For example, high levels of miR-10b-5p, miR-17-5p, miR-18a-5p, miR-19b-3p, miR-92a-3p, miR-125b-5p, miR-155-5p, miR-181a-5p, miR185-5p, miR-194-5p, miR-200c-3p, miR-215, and miR-372 in tumor tissues were found to be associated with unfavorable clinical outcomes; similarly, low levels of miR-16-5p, miR-22-3p, miR-93-5p, miR-106a-5p, miR-124-3p, miR126-3p, miR-128, miR-133b, miR-135b-5p miR-195-5p, miR-212-3p, and miR-362-3p were associated with worse survival (Table 2). In plasma, high levels of miR-140-5p, miR-141-3p, and miR-221-3p were associated with shorter OS, whereas low levels of miR-143-3p and miR-1224-5p predicted worse survival (Table 2). A major problem with the aforementioned miRNA marker studies (Table 2) is that many of the analyses were based on limited number of specimens and there was a lack of replication of the initial findings. Each study analyzed only a small number of cases, ranging from 24-273, with a median sample size of 89 . So far only four studies, ${ }^{12,20,26,87}$ which included $28,48,57$, and 193 cases, respectively, were prospective studies. The rest of the studies (Table 2) were either retrospective in nature or of uncertain study type. Retrospective study has disadvantages, such as selection bias and information bias. It is therefore impossible to rule out the likelihood of chance findings due to the nature of the study itself. Further prospective studies are warranted for validation of the prognostic power of the candidate miRNAs in CRC.

\section{miRNA processing machinery: DICER I}

RNase III endonuclease DICER1 performs a fundamental role in miRNA biogenesis by excising the stem-loop premiRNAs into functional miRNAs. Human DICER1 is an L-shaped 219-kilodalton multidomain protein including a DEAD-like helicase domain for double-stranded RNA translocation, a Piwi/Argonaute/Zwille domain for RNA-binding, a ruler domain, and a RNase III domain for double-stranded RNA cleavage. ${ }^{47}$ Unlike other organisms that have multiple Dicer proteins, DICER1 is the only form of Class 3 RNase III enzyme that is involved in both small interfering RNA (siRNA) and miRNA maturation in human cells. ${ }^{48}$ DICER1 is a haploinsufficient tumor suppressor, and deletion of DICER1 has been evidenced in various human cancers. ${ }^{49}$ Experimental evidence suggested that impaired DICER1 causes a global reduction in mature miRNA levels and promotes tumor growth and metastasis. ${ }^{49-51}$ Giving the central role of DICER1 in miRNA production, several studies have tried to evaluate the correlation between DICER1 level and its prognostic significance in CRC.

DICER1 is located on chromosome 14q32.13. A frequent loss of heterozygosity of this region was linked with metastatic recurrence of early-stage CRC. ${ }^{52}$ Akahane $^{53}$ evaluated the association between the expression levels of DICER 1 mRNA and the clinical outcomes in 260 CRC patients from Japan who did not receive any chemoradiotherapy prior to surgery. Based on laser microdissection and qRT-PCR, mRNA of DICER1 was significantly reduced in tumor compared to that in the adjacent normal tissue. Lower mRNA level of DICER1 was significantly associated with larger tumor size, greater invasion depth, more lymph node metastasis and lymphatic invasion, and more-advanced Dukes' stages. The OS and DFS of patients in the lower DICER1 group showed worse survival rates compared with the high DICER1 group. On the protein level, Faggad et $\mathrm{al}^{54}$ examined the expression of DICER1 in $331 \mathrm{CRC}$ patients by immunohistochemistry, of which 65 patients (19.6\%) showed a negative stain for DICER1. The mean OS time for the DICER1 negative group was 64.1 months, which was significantly shorter than in the positive group 


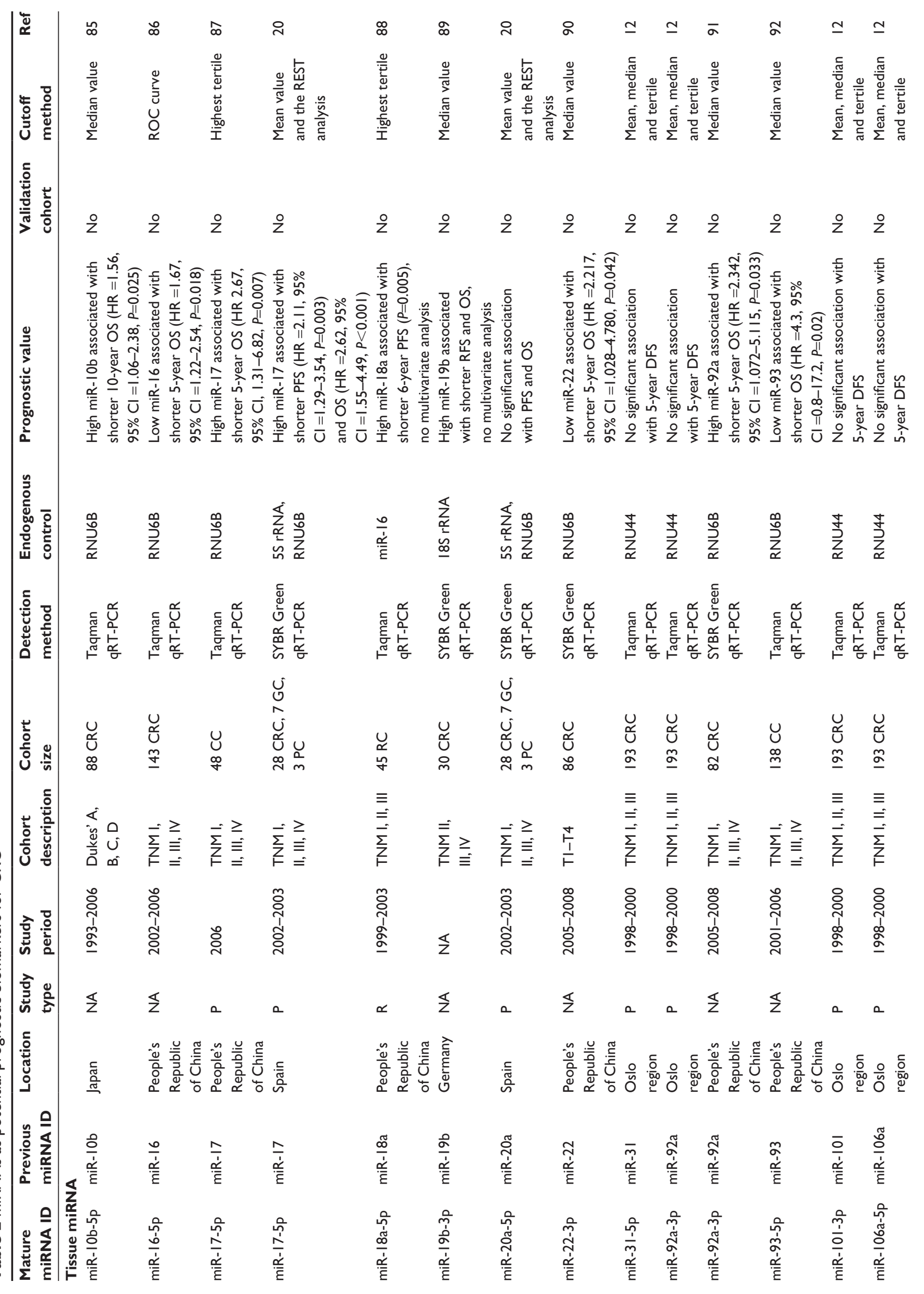




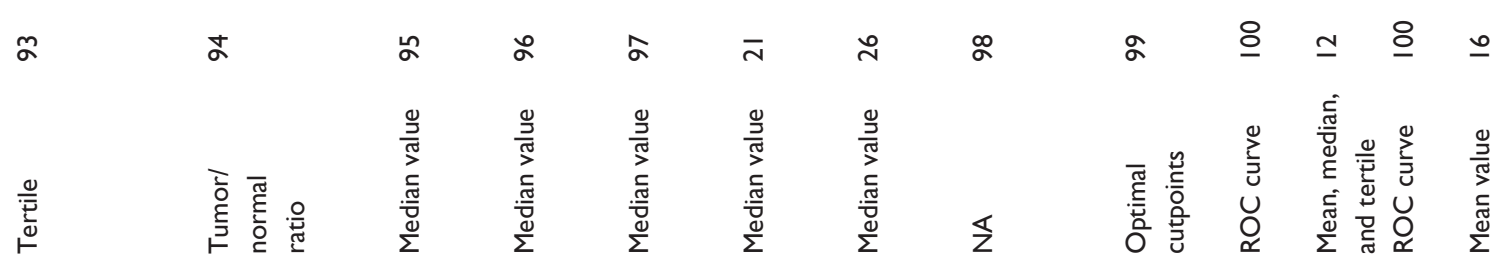

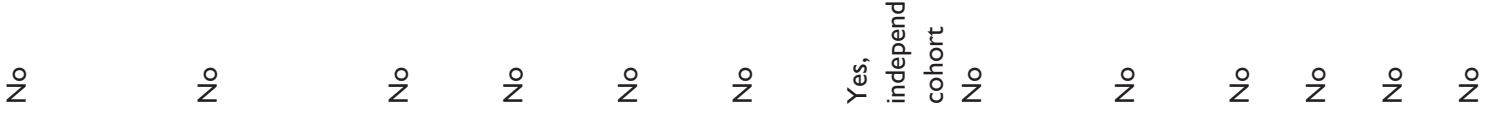

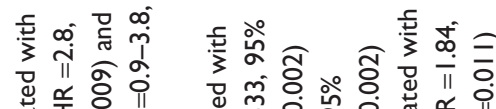

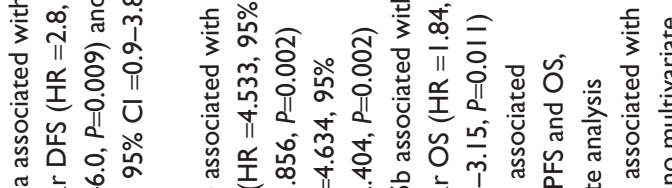

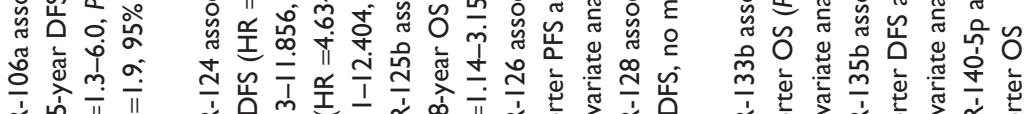

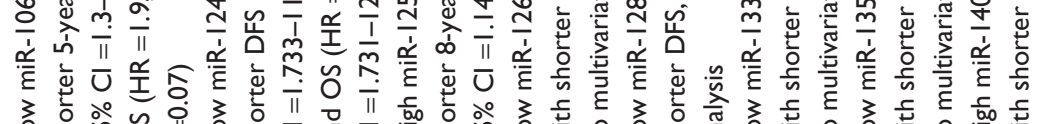

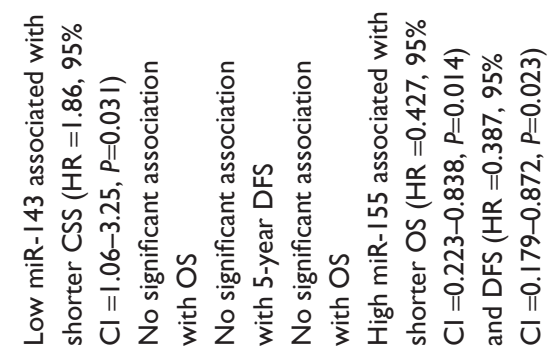

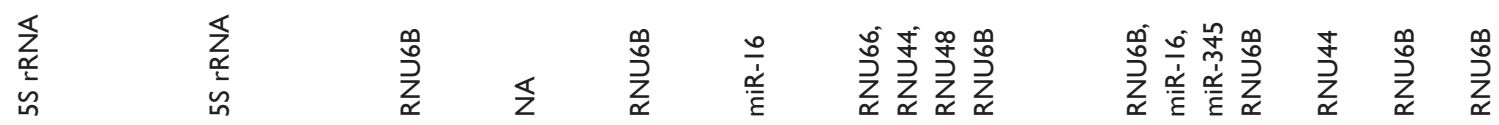

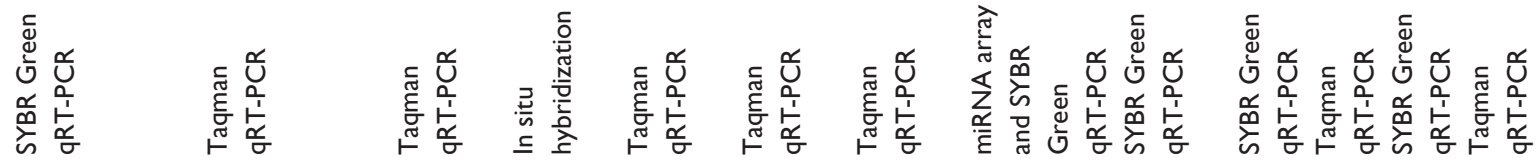

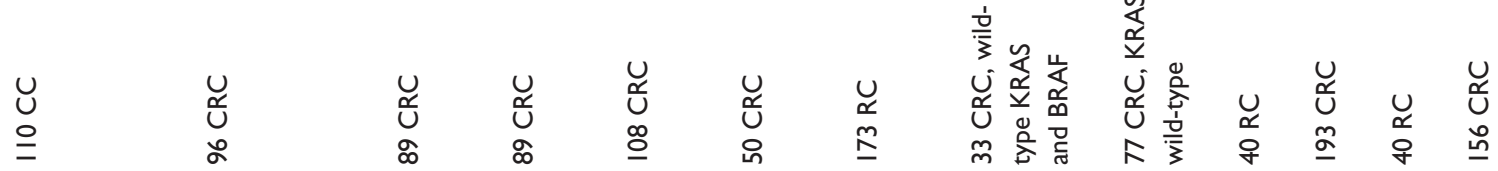

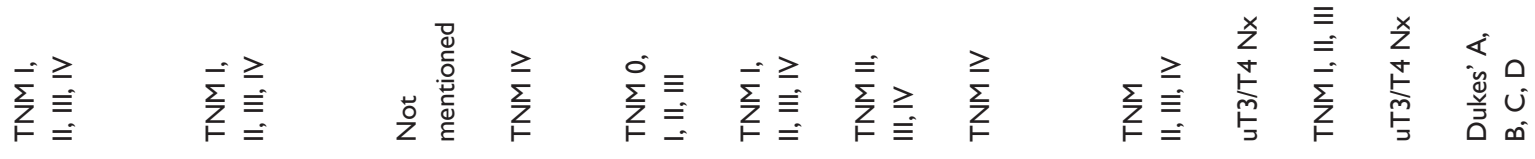

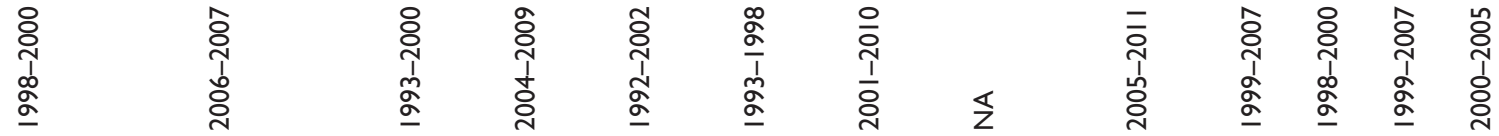

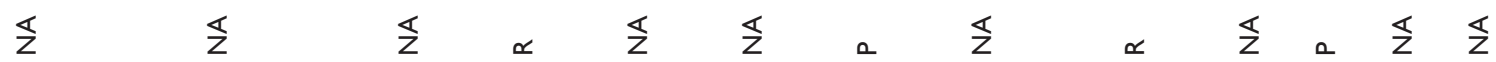

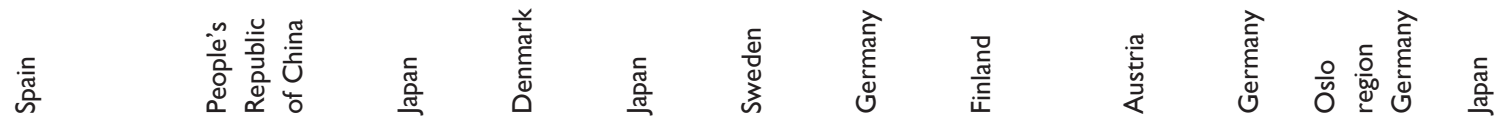

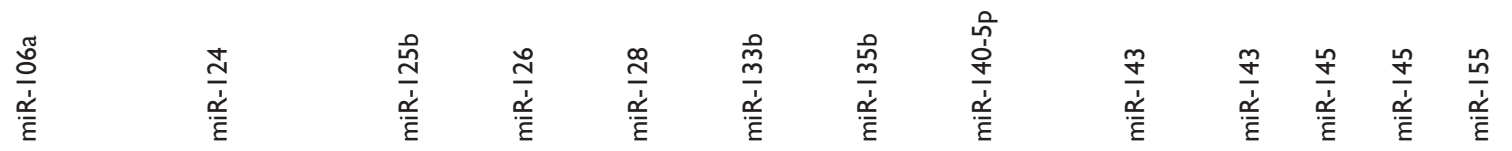

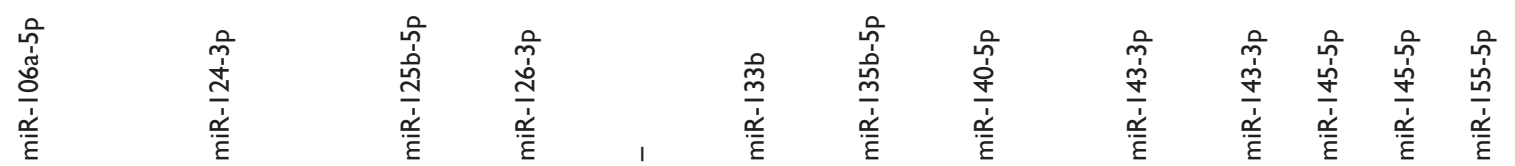




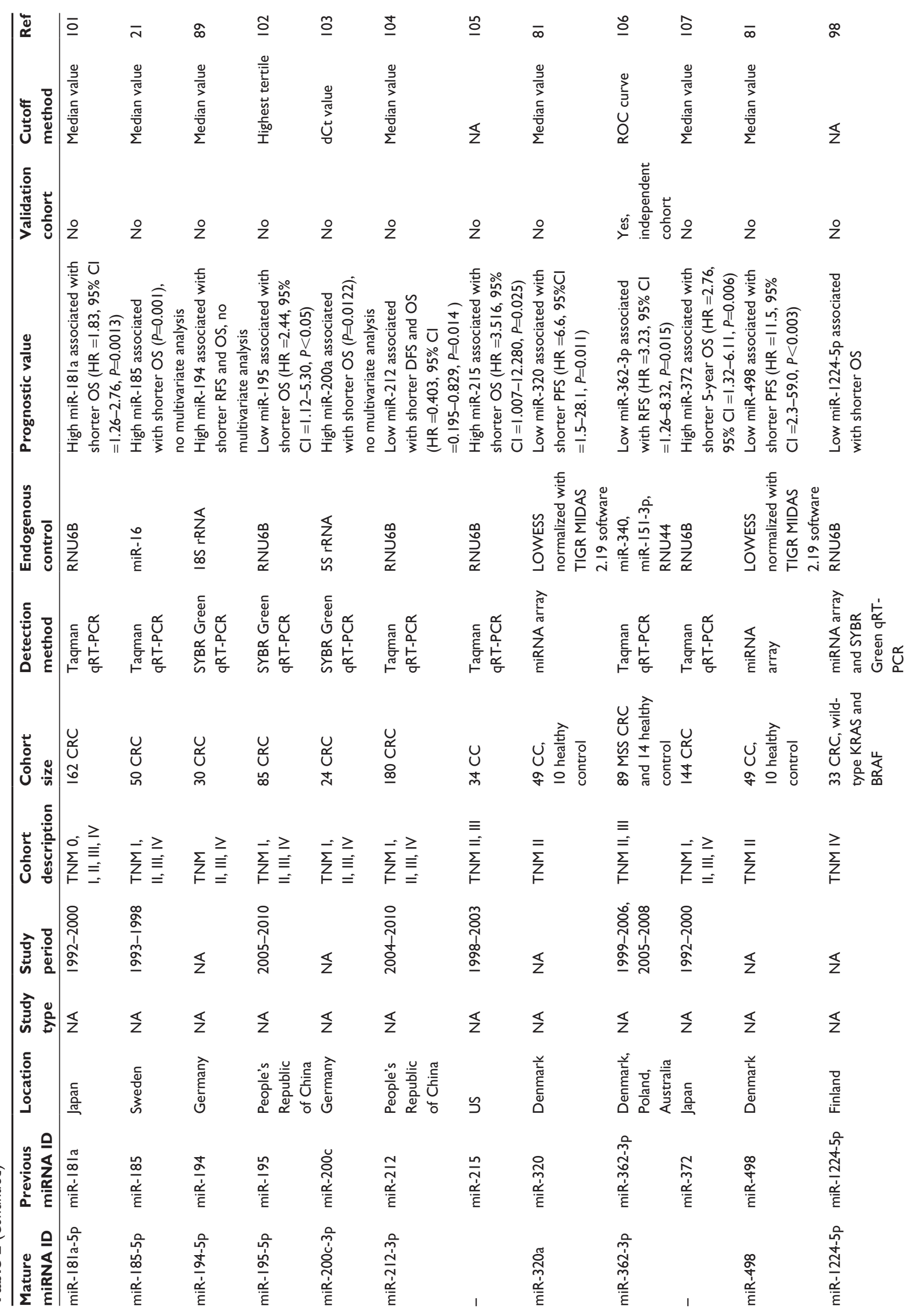




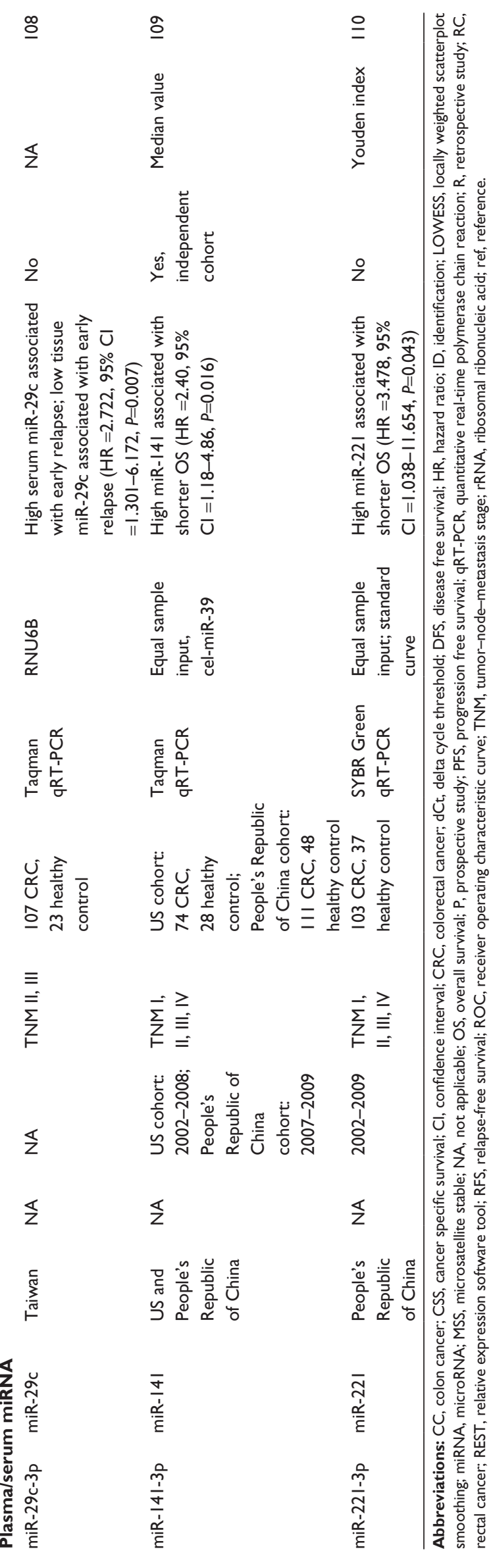

(88.6 months). ${ }^{53,54}$ Both of the studies supported DICER1 as an independent prognostic factor for OS.

These associations were challenged by other studies. Comparable expression of DICER1 was observed in primary CRC tissues and the corresponding normal mucosa in several independent studies. ${ }^{55-57}$ Faber et al ${ }^{58}$ examined 237 patients with moderately differentiated CRC by immunohistochemistry. The intense staining of DICER1 in CRC showed a strong association with poor cancer-specific survival and reduced PFS. Fifteen out of the 237 stage I/II CRCs were DICER1negative patients who did not experience any relapse in a 10-year follow-up, although the authors did not specify any correlation between DICER1 expression and tumor stage. ${ }^{58}$ Stratmann et al ${ }^{55}$ claimed that patients with high DICER1 mRNA expression in normal mucosa, but not in cancerous sites, were associated with worse clinical outcomes compared to those with a lower DICER1 expression.

\section{miRNA-associated single- nucleotide polymorphism}

The whole genome is constantly evolving and generates many germ-line single nucleotide alterations among individuals in a population. These alterations are known as single-nucleotide polymorphisms (SNPs). SNPs that locate in the protein-coding sequence may have an obvious biological effect by changing the amino acid sequence or yielding truncated protein product, whereas SNPs residing in the UTR do not alter the function of the protein, but they may occasionally perturb the protein expression level and may have pathogenic consequences. ${ }^{59}$ In 2006, a team of researchers first corroborated that a $\mathrm{G}$ to A substitution in the 3'UTR of GDF8 created an illegitimate miRNA octamer motif that could be transcriptionally downregulated by two miRNAs: miR-1-3p (miR-1) and miR-206. ${ }^{60}$ This discovery had promoted intensive research on the potential application of miRNA-associated polymorphisms as biomarkers for the clinical outcomes of cancer, especially the miRNA-related SNP on the $3^{\prime} \mathrm{UTR}$ of the KRAS gene.

The germline variation rs61764370 (also called let-7 miRNA complementary site, LCS6), located in the let-7 complementary site in the KRAS 3'UTR mRNA, is one of the most intensively studied polymorphism-associated miRNA target SNPs. Compared to the wild-type T genotype, the less-frequent variant $\mathrm{G}$ transcript of $K R A S$ exerts a high stability through escaping the let-7 translational repression and causes a high level of KRAS in the cell. ${ }^{61,62}$ Generally, Caucasians have a higher frequency of the $\mathrm{G}$ allele (17.2\%) compared to the other races. ${ }^{63}$ While the $\mathrm{G}$ allele frequency 
is comparable between healthy control, adenoma, and CRC, an increasing frequency is observed when the tumor stage increases, with $14 \%$ in the early stages and $21.4 \%-25.0 \%$ in the terminal stage. ${ }^{64-66}$ In 2010, Graziano et al ${ }^{66}$ first reported that the homozygous and heterozygous $G$ allele carriers exhibited a significantly worse PFS and OS than the wild-type TT genotype metastatic patients who carried a BRAF V600wildtype and received salvage cetuximab-irinotecan therapy. They also reported that, in a subgroup of 55 unresponsive patients carrying $K R A S$ mutation, G type carriers showed a median OS of 5.9 months and PFS of 2.5 months, which was significantly shorter than the TT genotype patients, who had a median OS of 9.7 months and PFS of 3.4 months. On the contrary, conflicting results were reported by Ryan et al. ${ }^{67}$ Based on a cohort of 237 cases of African-American and European American patients who were primarily treated with 5-fluorouracil, they found that the stage III/IV G allele carriers had a significantly reduced risk for death compared to the TT genotype, whereas no benefit was observed in the stage I and II subset. Smits et $\mathrm{al}^{65}$ observed that the G allele correlated with a lower mortality risk in stage I/II patients. Most recently, Sha et $\mathrm{al}^{63}$ carried out the largest cohort study to date and genotyped 2,834 stage III CC patients who received FOLFOX alone or combined with cetuximab. The variant-containing genotype showed no statistically significant association with DFS or time to recurrence in the whole cohort or in any treatment arm. Further, no correlations were observed between rs61764370 and molecular/clinical status, such as $K R A S, B R A F$, and mismatch repair, tumor grade, lymph-node status, and body mass index. In agreement with their findings, previous studies also suggested no association between rs61764370 and clinical outcomes of CRC, or stage IV CRC patients who were treated with Nordic FLOX, cetuximab, or both (Table 3). ${ }^{64,68,69}$ There is no clear explanation for the conflicting observations among studies. It is speculated that the chemotherapy backbone would be one confounding factor. ${ }^{64}$

A SNP presented in pri-, pre-, or mature miRNA itself or in the miRNA processing machinery will potentially affect the miRNA expression and function. ${ }^{70}$ Lin et $\mathrm{al}^{71}$ performed a very informative study. On the basis of data mining of several SNP datasets and an miRNA prediction algorithm, they selected 41 SNPs located in eleven genes related to miRNA biogenesis, and 15 in pri-, pre-, or mature miRNA sequences. In the training phase, after stratifying by stage, they found that $R A N /$ rs 14035 and miR-373/rs12983273 showed a highly significant association with recurrence-free survival in stage II patients, whereas miR-608/rs4919510, GEMIN3/ rs197412, XPO5/rs11077, AGO2/rs4961280, GEMIN4/ rs2740348, GEMIN3/rs197388, and GEMIN4/rs7813 did so in stage III patients. Among the 218 cases with stage IV disease, four SNPs (let-7f-2/rs17276588, miR-30c-1/rs16827546, DROSHA/rs6877842, and DICER1/rs13078) were linked with the risk for recurrence. For the OS, $A G O 2 / \mathrm{rs} 4961280$, miR-608/rs4919510, miR-219a-1 (miR-219-1)/rs213210, miR-604/rs2368392, DICER1/rs13078, and TRBP/rs784567 were associated with the risk of death. The authors further verified the prognostic power of the 16 SNPs, and two of them retained the strong association with stage III patients. In the independent validation cohort, training cohort, or the combined cohorts, the $\mathrm{C}>\mathrm{G}$ substitution in rs4919510 was associated with a higher risk for both recurrence and death, and a $\mathrm{C}>\mathrm{T}$ substitution in rs 213210 showed a significantly more adverse OS than the wild-type CC genotype. ${ }^{71}$ The SNP rs4919510 is located in the mature miR-608 sequence, whereas the functional consequence of miR-219a-1/rs 213210 is still unknown. It is speculated that rs213210 might affect the miR-219a-1 maturation. Lee et al ${ }^{72}$ validated all 16 SNPs identified in Lin's study, ${ }^{71}$ including miR-608/rs4919510 and miR-219a-1/rs213210. Unfortunately, none of the abovementioned SNPs retained the prognostic power in a Korean cohort. ${ }^{72}$ Intriguingly, a completely opposite clinical outcome and result for miR-608/rs4919510 was observed in a Chinese Han population. ${ }^{73}$ Xing et $\mathrm{al}^{73}$ found that the $\mathrm{G}$ allele carriers had a significantly favorable recurrence-free survival than the CC wild-type. Moreover, the association between rs4919510 and clinical outcome was more prominent in a subset of patients who received chemotherapy.

SNP rs2910164 resides in the stem region opposite to the mature miR-146a-5p (miR-146a). Experimental evidence demonstrated that the presence of the rare $\mathrm{C}$ allele caused $\mathrm{a}$ less-efficient processing reaction in vitro and ultimately led to a decreased level of mature miR-146a-5p. ${ }^{74}$ Although the biological function of miR-146a-5p in CRC progression is still unknown, previous studies suggested that miR-146a-5p could negatively regulate the immune response. ${ }^{75}$ Chae et al ${ }^{76}$ observed that the GG or GC genotypes of rs2910164 were associated with better relapse-free and disease-specific survival compared with the homozygote CC genotype. However, in another Korea-based study, rs2910164 was shown to have no association with OS or relapse-free survival. ${ }^{77}$

\section{miRNA and microsatellite instability}

CRC mainly arises through two distinct mutational pathways. The first pathway is chromosomal instability characterized 


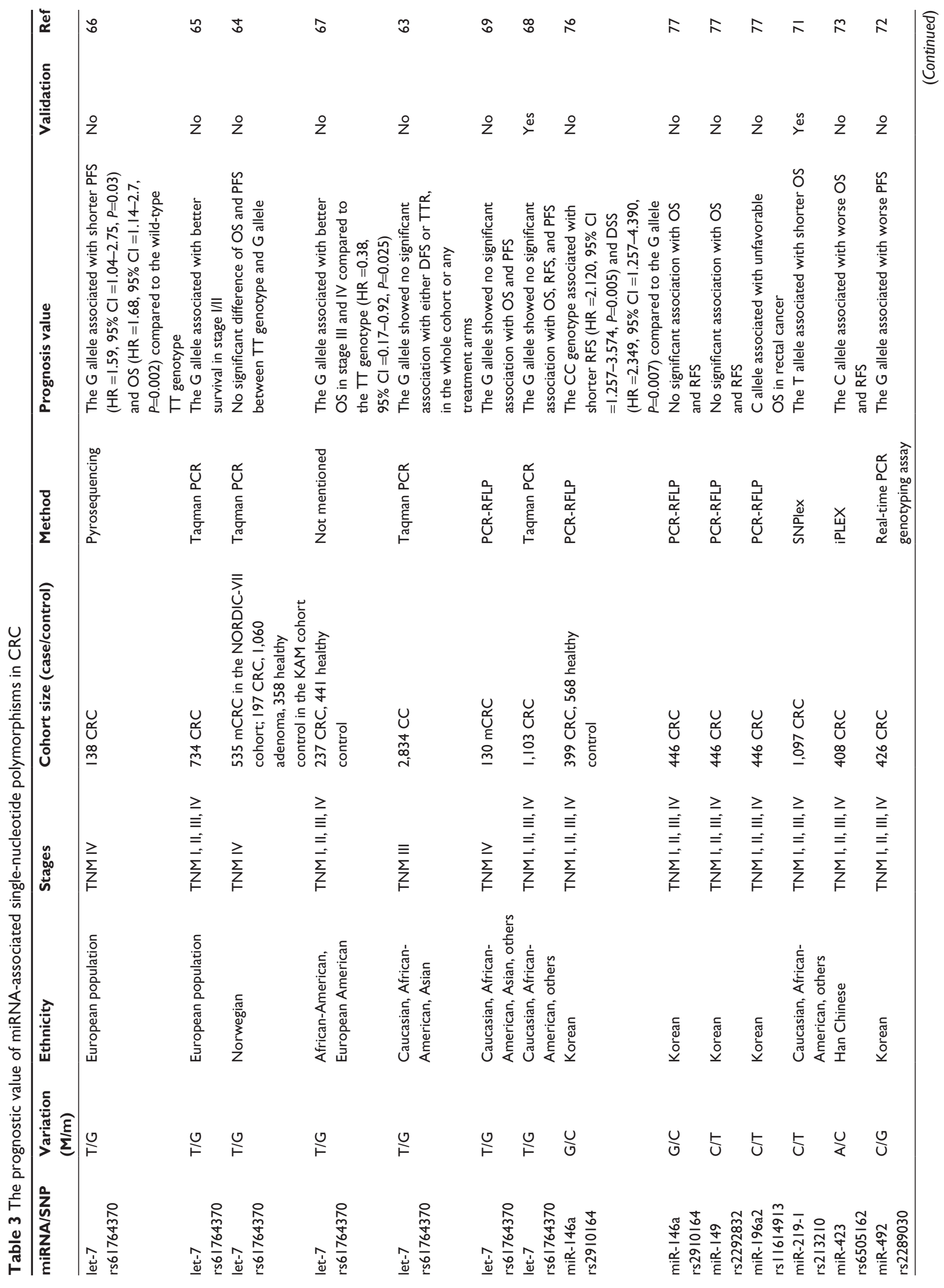




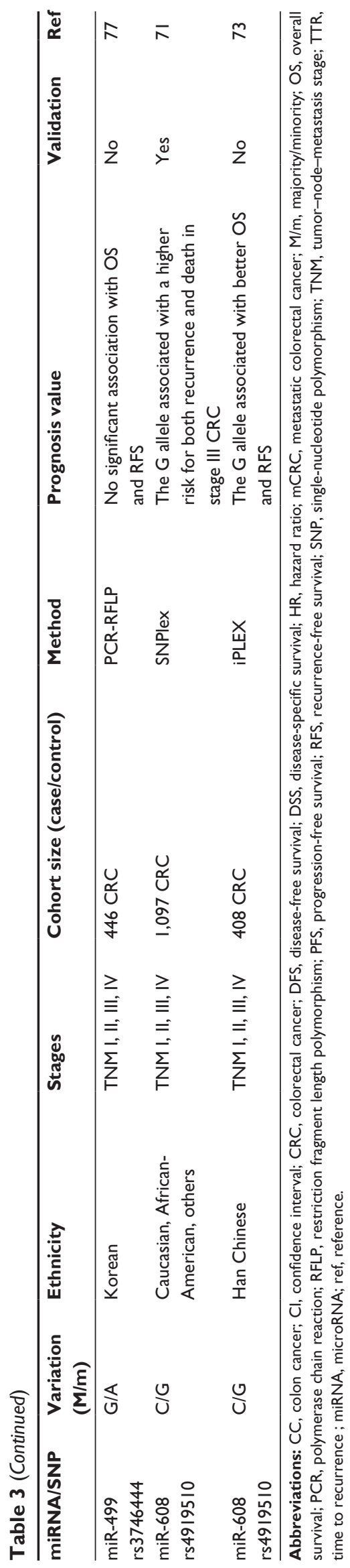

by an imbalance in chromosome number, subchromosomal genomic amplifications, and a high frequency of loss of heterozygosity. ${ }^{78}$ The other pathway is the MSI pathway, featured by increased short tandem repeats (microsatellites) due to a malfunctioning DNA mismatch repair system, and it accounts for $15 \%$ of all cases of CRC. ${ }^{79}$ Although MSI and microsatellite stable (MSS) are two histologically similar CRC subtypes, they have different clinical and pathologic features. In general, MSI patients have better survival and are less likely to develop metastasis. ${ }^{79} \mathrm{It}$ is therefore assumed that MSI-related miRNAs have prognostic potential as well.

Indeed, it has been proven that CRC tumors have different miRNA expression signatures according to their MSI status. ${ }^{80-83}$ Lanza et al firstly reported a list of 27 predictors of $\mathrm{mRNA} / \mathrm{miRNA}$ that can discriminate MSI-high (MSI-H) from MSS tumors. ${ }^{80}$ Schepeler et al ${ }^{81}$ focused on MSI-related miRNA profiles in a study of 49 patients with stage II CC. They identified a four-miRNA-signature (miR-142-3p, miR212-3p [miR-212], miR-151a-3p [miR-151], and miR-144-3p [miR-144]) that can specifically discriminate stage II CC according to microsatellite status. Sarver et al ${ }^{82}$ dichotomized 80 subjects into sporadic MSI-H group and MSS/MSI-low (MSI-L) group. They revealed that four miRNAs (miR-552, miR-592, miR-181c-5p [miR-181c], and miR-196b-5p [miR196b]) were decreased in MSS/MSI-L patients compared with the MSI-H group, whereas miR-625 and miR-31 exhibited increased expression in MSI-H group. In addition to the sporadic MSI cases, Balaguer et $\mathrm{al}^{83}$ included hereditary nonpolyposis $\mathrm{CC}$ cases in their study. They demonstrated that a signature of 59 miRNAs was able to distinguish MSI from MSS tumors. Moreover, they reported that an miRNA signature (miR-622, miR-362-5p, and miR-486-5p) was able to accurately discriminate hereditary nonpolyposis colorectal cancer cases from sporadic MSI patients. Earle et a ${ }^{84}$ selected 23 miRNAs' based on previous work and evaluated these miRNAs' expression in a cohort of 55 CRC cases. They characterized the study cohort as MSI-H, MSI-L, and MSS as determined by microsatellite marker polymerase chain reaction or immunohistochemistry. Elevated relative expression of miR-155-5p (miR-155), miR-31-5p (miR-31), miR-223-3p (miR-223), and miR-26b-5p (miR-26b) was significantly associated with MSI-H status, whereas increased relative expression of miR-92a-3p (miR-92), let-7a-5p (let-7a), and miR-145-5p (miR-145) was associated with MSI-L. Increased relative expression of miR-196a was associated with MSS status. Five independent studies ${ }^{80-84}$ described MSI-associated candidate miRNAs, but only part of the candidates overlapped with each other (eg, miR-155-5p and miR-223-3p). 
Furthermore, they have seldom been validated at the MSI/ MSS background. Finally, caution has to be taken when interpreting these MSI-related miRNA markers. For example, increased miR-155-5p was identified as a MSI-H marker, which theoretically should be regarded as a favorable prognostic factor. ${ }^{80,84}$ On the other hand, high tissue miR-155-5p was observed to be associated with lymph-node metastasis and independently predicted higher risk for mortality. ${ }^{16}$

\section{Conclusion and future perspectives}

In this article, we have introduced the recent findings concerning the prognostic potential of miRNAs in CRC. Although the literature of identification of novel miRNA markers has increased rapidly in the last 7 years, we are still in the very initial stage of the clinical-application realm. So far, three tissue miRNAs (miR-21-5p, miR-29-3p, miR$148-3 p$ ) have been examined in multiple studies, of which miR-21-5p is the most promising prognostic marker, yet further prospective validation studies are required before it can go into clinical use. Most of the current research comprises initial exploratory studies that suffered from methodologic flaws, including small sample size, nontransparent patient information, lack of replication, and poor statistical analysis. We are expecting a multimarker signature in the future that can accurately predict clinical outcomes, although the costefficiency issue should be also considered. Moreover, for each potential prognostic biomarker, it is necessary to understand its molecular function and the associated mechanisms behind its dysregulation, which may help support its clinical use and provide novel therapeutic targets.

\section{Acknowledgment}

This study was funded by the General Research Fund Hong Kong (472613).

\section{Disclosure}

The authors report no conflicts of interest in this work.

\section{References}

1. Jemal A, Bray F, Center MM, Ferlay J, Ward E, Forman D. Global cancer statistics. CA Cancer J Clin. 2011;61(2):69-90.

2. Sung JJ, Lau JY, Young GP, et al; Asia Pacific Working Group on Colorectal Cancer. Asia Pacific consensus recommendations for colorectal cancer screening. Gut. 2008;57(8):1166-1176.

3. Wolpin BM, Mayer RJ. Systemic treatment of colorectal cancer. Gastroenterology. 2008;134(5):1296-1310.

4. Wang Y, Jatkoe T, Zhang Y, et al. Gene expression profiles and molecular markers to predict recurrence of Dukes' B colon cancer. J Clin Oncol. 2004;22(9):1564-1571.

5. Bartel DP. MicroRNAs: genomics, biogenesis, mechanism, and function. Cell. 2004;116(2):281-297.
6. Friedman RC, Farh KK, Burge CB, Bartel DP. Most mammalian mRNAs are conserved targets of microRNAs. Genome Res. 2009;19(1):92-105.

7. Lu J, Getz G, Miska EA, et al. MicroRNA expression profiles classify human cancers. Nature. 2005;435(7043):834-838.

8. Volinia S, Calin GA, Liu CG, et al. A microRNA expression signature of human solid tumors defines cancer gene targets. Proc Natl Acad Sci U S A. 2006;103(7):2257-2261.

9. Dong Y, Wu WK, Wu CW, Sung JJ, Yu J, Ng SS. MicroRNA dysregulation in colorectal cancer: a clinical perspective. $\mathrm{Br} J$ Cancer. 2011;104(6):893-898.

10. Wu WK, Law PT, Lee CW, et al. MicroRNA in colorectal cancer: from benchtop to bedside. Carcinogenesis. 2011;32(3):247-253.

11. de Krijger I, Mekenkamp LJ, Punt CJ, Nagtegaal ID. MicroRNAs in colorectal cancer metastasis. J Pathol. 2011;224(4):438-447.

12. Schee K, Boye K, Abrahamsen TW, Fodstad Ø, Flatmark K. Clinical relevance of microRNA miR-21, miR-31, miR-92a, miR-101, miR-106a and miR-145 in colorectal cancer. BMC Cancer. 2012;12:505.

13. Schee K, Lorenz S, Worren MM, et al. Deep sequencing the microRNA transcriptome in colorectal cancer. PLoS One. 2013;8(6):e66165.

14. Schetter AJ, Leung SY, Sohn JJ, et al. MicroRNA expression profiles associated with prognosis and therapeutic outcome in colon adenocarcinoma. JAMA. 2008;299(4):425-436.

15. Nielsen BS, Jørgensen S, Fog JU, et al. High levels of microRNA-21 in the stroma of colorectal cancers predict short disease-free survival in stage II colon cancer patients. Clin Exp Metastasis. 2011;28(1): $27-38$.

16. Shibuya $H$, Iinuma $H$, Shimada $R$, Horiuchi A, Watanabe T. Clinicopathological and prognostic value of microRNA-21 and microRNA-155 in colorectal cancer. Oncology. 2010;79(3-4):313-320.

17. Schetter AJ, Nguyen GH, Bowman ED, et al. Association of inflammation-related and microRNA gene expression with cancerspecific mortality of colon adenocarcinoma. Clin Cancer Res. 2009;15(18):5878-5887.

18. Zhang JX, Song W, Chen ZH, et al. Prognostic and predictive value of a microRNA signature in stage II colon cancer: a microRNA expression analysis. Lancet Oncol. 2013;14(13):1295-1306.

19. Kulda V, Pesta M, Topolcan O, et al. Relevance of miR-21 and miR143 expression in tissue samples of colorectal carcinoma and its liver metastases. Cancer Genet Cytogenet. 2010;200(2):154-160.

20. Valladares-Ayerbes M, Blanco M, Haz M, et al. Prognostic impact of disseminated tumor cells and microRNA-17-92 cluster deregulation in gastrointestinal cancer. Int J Oncol. 2011;39(5):1253-1264.

21. Akçakaya P, Ekelund S, Kolosenko I, et al. miR-185 and miR-133b deregulation is associated with overall survival and metastasis in colorectal cancer. Int J Oncol. 2011;39(2):311-318.

22. Liu GH, Zhou ZG, Chen R, et al. Serum miR-21 and miR-92a as biomarkers in the diagnosis and prognosis of colorectal cancer. Tumour Biol. 2013;34(4):2175-2181.

23. Menéndez P, Padilla D, Villarejo P, et al. Prognostic implications of serum microRNA-21 in colorectal cancer. J Surg Oncol. 2013;108(6): 369-373.

24. Slaby O, Svoboda M, Fabian P, et al. Altered expression of miR-21, miR-31, miR-143 and miR-145 is related to clinicopathologic features of colorectal cancer. Oncology. 2007;72(5-6):397-402.

25. Tibshirani R. Regression shrinkage and selection via the lasso. J R Statist Soc B. 1996;58(1):267-288.

26. Gaedcke J, Grade M, Camps J, et al. The rectal cancer microRNAomemicroRNA expression in rectal cancer and matched normal mucosa. Clin Cancer Res. 2012;18(18):4919-4930.

27. Slattery ML, Wolff E, Hoffman MD, Pellatt DF, Milash B, Wolff RK. MicroRNAs and colon and rectal cancer: differential expression by tumor location and subtype. Genes Chromosomes Cancer. 2011;50(3): 196-206.

28. Yamamichi N, Shimomura R, Inada K, et al. Locked nucleic acid in situ hybridization analysis of miR-21 expression during colorectal cancer development. Clin Cancer Res. 2009;15(12):4009-4016. 
29. Bullock MD, Pickard KM, Nielsen BS, et al. Pleiotropic actions of miR-21 highlight the critical role of deregulated stromal microRNAs during colorectal cancer progression. Cell Death Dis. 2013;4:e684.

30. Bullock M, Pickard K, Nielsen BS, et al. Deregulated stromal microRNA-21 and promotion of metastatic progression in colorectal cancer. Proceedings of the Spring Meeting for Clinician Scientists in Training, Academy of Medical Sciences' Annual Spring Meeting; February 26, 2014: London, UK. Lancet. 2014;383:S30.

31. Fu J, Tang W, Du P, et al. Identifying microRNA-mRNA regulatory network in colorectal cancer by a combination of expression profile and bioinformatics analysis. BMC Syst Biol. 2012;6:68.

32. Bandrés E, Cubedo E, Agirre $X$, et al. Identification by Real-time PCR of 13 mature microRNAs differentially expressed in colorectal cancer and non-tumoral tissues. Mol Cancer. 2006;5:29.

33. Arndt GM, Dossey L, Cullen LM, et al. Characterization of global microRNA expression reveals oncogenic potential of miR-145 in metastatic colorectal cancer. BMC Cancer. 2009;9:374.

34. Slattery ML, Wolff E, Hoffman MD, Pellatt DF, Milash B, Wolff RK. MicroRNAs and colon and rectal cancer: differential expression by tumor location and subtype. Genes Chromosomes Cancer. 2011;50(3):196-206.

35. Huang Z, Huang D, Ni S, Peng Z, Sheng W, Du X. Plasma microRNAs are promising novel biomarkers for early detection of colorectal cancer. Int J Cancer. 2010;127(1):118-126.

36. Brunet Vega A, Pericay C, Moya I, et al. microRNA expression profile in stage III colorectal cancer: circulating miR-18a and miR-29a as promising biomarkers. Oncol Rep. 2013;30(1):320-326.

37. Wang LG, Gu J. Serum microRNA-29a is a promising novel marker for early detection of colorectal liver metastasis. Cancer Epidemiol. 2012;36(1):e61-e67.

38. Tang W, Zhu Y, Gao J, et al. MicroRNA-29a promotes colorectal cancer metastasis by regulating matrix metalloproteinase 2 and E-cadherin via KLF4. Br J Cancer. 2014;110(2):450-458.

39. Croce CM, Harris CC, Schetter AJ, inventors. Method of diagnosing poor survival prognosis colon cancer using mir-29a. United States Patent US 8338106 B2. December 25, 2012.

40. Faltejskova P, Bocanek O, Sachlova M, et al. Circulating miR-17-3p, miR-29a, miR-92a and miR-135b in serum: Evidence against their usage as biomarkers in colorectal cancer. Cancer Biomark. 2012;12(4):199-204.

41. Weissmann-Brenner A, Kushnir M, Lithwick Yanai G, et al. Tumor microRNA-29a expression and the risk of recurrence in stage II colon cancer. Int J Oncol. 2012;40(6):2097-2103.

42. Lee $Y$, Yang X, Huang Y, et al. Network modeling identifies molecular functions targeted by miR-204 to suppress head and neck tumor metastasis. PLoS Comput Biol. 2010;6(4):e1000730.

43. Kuo TY, Hsi E, Yang IP, Tsai PC, Wang JY, Juo SH. Computational analysis of mRNA expression profiles identifies microRNA-29a/c as predictor of colorectal cancer early recurrence. PLoS One. 2012;7(2):e31587.

44. Chen Y, Song Y, Wang Z, et al. Altered expression of MiR-148a and MiR-152 in gastrointestinal cancers and its clinical significance. J Gastrointest Surg. 2010;14(7):1170-1179.

45. Takahashi M, Cuatrecasas M, Balaguer F, et al. The clinical significance of MiR-148a as a predictive biomarker in patients with advanced colorectal cancer. PLoS One. 2012;7(10):e46684.

46. Tsai HL, Yang IP, Huang CW, et al. Clinical significance of microRNA148 a in patients with early relapse of stage II stage and III colorectal cancer after curative resection. Transl Res. 2013;162(4):258-268.

47. Lau PW, Guiley KZ, De N, Potter CS, Carragher B, MacRae IJ. The molecular architecture of human Dicer. Nat Struct Mol Biol. 2012;19(4):436-440.

48. Carthew RW, Sontheimer EJ. Origins and mechanisms of miRNAs and siRNAs. Cell. 2009;136(4):642-655.

49. Kumar MS, Pester RE, Chen CY, et al. Dicer1 functions as a haploinsufficient tumor suppressor. Genes Dev. 2009;23(23):2700-2704.
50. Kumar MS, Lu J, Mercer KL, Golub TR, Jacks T. Impaired microRNA processing enhances cellular transformation and tumorigenesis. Nat Genet. 2007;39(5):673-677.

51. Iliou MS, da Silva-Diz V, Carmona FJ, et al. Impaired DICER1 function promotes stemness and metastasis in colon cancer. Oncogene. 2014;33(30):4003-4015.

52. Al-Mulla F, AlFadhli S, Al-Hakim AH, Going JJ, Bitar MS. Metastatic recurrence of early-stage colorectal cancer is linked to loss of heterozygosity on chromosomes 4 and 14q. J Clin Pathol. 2006;59(6): 624-630.

53. Akahane T. Clinicopathological and prognostic significance of the microRNA processing enzyme DICER1 mRNA expression in colorectal cancer patients. Mol Clin Oncol. 2013;1(2):267-273.

54. Faggad A, Kasajima A, Weichert W, et al. Down-regulation of the microRNA processing enzyme Dicer is a prognostic factor in human colorectal cancer. Histopathology. 2012;61(4):552-561.

55. Stratmann J, Wang CJ, Gnosa S, et al. Dicer and miRNA in relation to clinicopathological variables in colorectal cancer patients. BMC Cancer. 2011;11:345.

56. Chiosea S, Acquafondata M, Luo J, Kuan S, Seethala R. DICER1 and PRKRA in colon adenocarcinoma. Biomark Insights. 2008;3:253-258.

57. Papachristou DJ, Korpetinou A, Giannopoulou E, et al. Expression of the ribonucleases Drosha, Dicer, and Ago2 in colorectal carcinomas. Virchows Arch. 2011;459(4):431-440.

58. Faber C, Horst D, Hlubek F, Kirchner T. Overexpression of Dicer predicts poor survival in colorectal cancer. Eur J Cancer. 2011;47(9): 1414-1419.

59. Yu Z, Li Z, Jolicoeur N, et al. Aberrant allele frequencies of the SNPs located in microRNA target sites are potentially associated with human cancers. Nucleic Acids Res. 2007;35(13):4535-4541.

60. Clop A, Marcq F, Takeda H, et al. A mutation creating a potential illegitimate microRNA target site in the myostatin gene affects muscularity in sheep. Nat Genet. 2006;38(7):813-818.

61. Johnson SM, Grosshans H, Shingara J, et al. RAS is regulated by the let-7 microRNA family. Cell. 2005;120(5):635-647.

62. Chin LJ, Ratner E, Leng S, et al. A SNP in a let-7 microRNA complementary site in the KRAS $3^{\prime}$ untranslated region increases non-small cell lung cancer risk. Cancer Res. 2008;68(20):8535-8540.

63. Sha D, Lee AM, Shi Q, et al. Association study of the let-7 miRNAcomplementary site variant in the $3^{\prime}$ untranslated region of the KRAS gene in stage III colon cancer (NCCTG N0147 Clinical Trial). Clin Cancer Res. 2014;20(12):3319-3327.

64. Kjersem JB, Ikdahl T, Guren T, et al. Let-7 miRNA-binding site polymorphism in the KRAS $3^{\prime}$ UTR; colorectal cancer screening population prevalence and influence on clinical outcome in patients with metastatic colorectal cancer treated with 5-fluorouracil and oxaliplatin \pm cetuximab. BMC Cancer. 2012;12:534.

65. Smits KM, Paranjape T, Nallur S, et al. A let-7 microRNA SNP in the KRAS 3'UTR is prognostic in early-stage colorectal cancer. Clin Cancer Res. 2011;17(24):7723-7731.

66. Graziano F, Canestrari E, Loupakis F, et al. Genetic modulation of the Let-7 microRNA binding to KRAS 3'-untranslated region and survival of metastatic colorectal cancer patients treated with salvage cetuximabirinotecan. Pharmacogenomics J. 2010;10(5):458-464.

67. Ryan BM, Robles AI, Harris CC. KRAS-LCS6 genotype as a prognostic marker in early-stage CRC - letter. Clin Cancer Res. 2012;18(12):3487-8; author reply 3489.

68. Ye Y, Madison B, Wu X, Rustgi AK. A LIN28B polymorphism predicts for colon cancer survival. Cancer Biol Ther. 2012;13(14): 1390-1395.

69. Zhang W, Winder T, Ning Y, et al. A let-7 microRNA-binding site polymorphism in 3'-untranslated region of KRAS gene predicts response in wild-type KRAS patients with metastatic colorectal cancer treated with cetuximab monotherapy. Ann Oncol. 2011;22(1):104-109.

70. Ryan BM, Robles AI, Harris CC. Genetic variation in microRNA networks: the implications for cancer research. Nat Rev Cancer. 2010;10(6):389-402. 
71. Lin M, Gu J, Eng C, et al. Genetic polymorphisms in MicroRNA-related genes as predictors of clinical outcomes in colorectal adenocarcinoma patients. Clin Cancer Res. 2012;18(14):3982-3991.

72. Lee HC, Kim JG, Chae YS, et al. Prognostic impact of microRNArelated gene polymorphisms on survival of patients with colorectal cancer. J Cancer Res Clin Oncol. 2010;136(7):1073-1078.

73. Xing J, Wan S, Zhou F, et al. Genetic polymorphisms in pre-microRNA genes as prognostic markers of colorectal cancer. Cancer Epidemiol Biomarkers Prev. 2012;21(1):217-227.

74. Jazdzewski K, Murray EL, Franssila K, Jarzab B, Schoenberg DR, de la Chapelle A. Common SNP in pre-miR-146a decreases mature $\mathrm{miR}$ expression and predisposes to papillary thyroid carcinoma. Proc Natl Acad Sci U S A. 2008;105(20):7269-7274.

75. O'Connell RM, Rao DS, Baltimore D. microRNA regulation of inflammatory responses. Annu Rev Immunol. 2012;30:295-312.

76. Chae YS, Kim JG, Lee SJ, et al. A miR-146a polymorphism (rs2910164) predicts risk of and survival from colorectal cancer. Anticancer Res. 2013;33(8):3233-3239.

77. Jang MJ, Kim JW, Min KT, Jeon YJ, Oh D, Kim NK. Prognostic significance of microRNA gene polymorphisms in patients with surgically resected colorectal cancer. Exp Ther Med. 2011;2(6):1127-1132.

78. Pino MS, Chung DC. The chromosomal instability pathway in colon cancer. Gastroenterology. 2010;138(6):2059-2072.

79. Gryfe R, Kim H, Hsieh ET, et al. Tumor microsatellite instability and clinical outcome in young patients with colorectal cancer. $N$ Engl J Med. 2000;342(2):69-77.

80. Lanza G, Ferracin M, Gafà R, et al. mRNA/microRNA gene expression profile in microsatellite unstable colorectal cancer. Mol Cancer. 2007;6:54

81. Schepeler T, Reinert JT, Ostenfeld MS, et al. Diagnostic and prognostic microRNAs in stage II colon cancer. Cancer Res. 2008;68(15): 6416-6424.

82. Sarver AL, French AJ, Borralho PM, et al. Human colon cancer profiles show differential microRNA expression depending on mismatch repair status and are characteristic of undifferentiated proliferative states. $B M C$ Cancer. 2009;9:401.

83. Balaguer F, Moreira L, Lozano JJ, et al. Colorectal cancers with microsatellite instability display unique miRNA profiles. Clin Cancer Res. 2011;17(19):6239-6249.

84. Earle JS, Luthra R, Romans A, et al. Association of microRNA expression with microsatellite instability status in colorectal adenocarcinoma. J Mol Diagn. 2010;12(4):433-440.

85. Nishida N, Yamashita S, Mimori K, et al. MicroRNA-10b is a prognostic indicator in colorectal cancer and confers resistance to the chemotherapeutic agent 5-fluorouracil in colorectal cancer cells. Ann Surg Oncol. 2012;19(9):3065-3071.

86. Qian J, Jiang B, Li M, Chen J, Fang M. Prognostic significance of microRNA-16 expression in human colorectal cancer. World J Surg. 2013;37(12):2944-2949.

87. Yu G, Tang JQ, Tian ML, et al. Prognostic values of the miR-17-92 cluster and its paralogs in colon cancer. J Surg Oncol. 2012;106(3):232-237.

88. Wu CW, Dong YJ, Liang QY, et al. MicroRNA-18a attenuates DNA damage repair through suppressing the expression of ataxia telangiectasia mutated in colorectal cancer. PLoS One. 2013;8(2):e57036.

89. Kahlert C, Klupp F, Brand K, et al. Invasion front-specific expression and prognostic significance of microRNA in colorectal liver metastases. Cancer Sci. 2011;102(10):1799-1807.

90. Zhang G, Xia S, Tian H, Liu Z, Zhou T. Clinical significance of miR-22 expression in patients with colorectal cancer. Med Oncol. 2012;29(5):3108-3112.

91. Zhou T, Zhang G, Liu Z, Xia S, Tian H. Overexpression of miR-92a correlates with tumor metastasis and poor prognosis in patients with colorectal cancer. Int J Colorectal Dis. 2013;28(1):19-24.
92. Xiao ZG, Deng ZS, Zhang YD, Zhang Y, Huang ZC. Clinical significance of microRNA-93 downregulation in human colon cancer. Eur J Gastroenterol Hepatol. 2013;25(3):296-301.

93. Díaz R, Silva J, García JM, et al. Deregulated expression of miR-106a predicts survival in human colon cancer patients. Genes Chromosomes Cancer. 2008;47(9):794-802.

94. Wang MJ, Li Y, Wang R, et al. Downregulation of microRNA-124 is an independent prognostic factor in patients with colorectal cancer. Int J Colorectal Dis. 2013;28(2):183-189.

95. Nishida N, Yokobori T, Mimori K, et al. MicroRNA miR-125b is a prognostic marker in human colorectal cancer. Int J Oncol. 2011;38(5): 1437-1443

96. Hansen TF, Sørensen FB, Lindebjerg J, Jakobsen A. The predictive value of microRNA-126 in relation to first line treatment with capecitabine and oxaliplatin in patients with metastatic colorectal cancer. BMC Cancer. 2012;12:83.

97. Takahashi Y, Iwaya T, Sawada G, et al. Up-regulation of NEK2 by microRNA-128 methylation is associated with poor prognosis in colorectal cancer. Ann Surg Oncol. 2014;21(1):205-212.

98. Mosakhani N, Lahti L, Borze I, et al. MicroRNA profiling predicts survival in anti-EGFR treated chemorefractory metastatic colorectal cancer patients with wild-type KRAS and BRAF. Cancer Genet. 2012;205(11):545-551.

99. Pichler M, Winter E, Stotz M, et al. Down-regulation of KRASinteracting miRNA-143 predicts poor prognosis but not response to EGFR-targeted agents in colorectal cancer. Br J Cancer. 2012;106(11): 1826-1832.

100. Drebber U, Lay M, Wedemeyer I, et al. Altered levels of the onco-microRNA 21 and the tumor-supressor microRNAs 143 and 145 in advanced rectal cancer indicate successful neoadjuvant chemoradiotherapy. Int J Oncol. 2011;39(2):409-415.

101. Nishimura J, Handa R, Yamamoto H, et al. microRNA-181a is associated with poor prognosis of colorectal cancer. Oncol Rep. 2012;28(6):2221-2226.

102. Wang X, Wang J, Ma H, Zhang J, Zhou X. Downregulation of miR-195 correlates with lymph node metastasis and poor prognosis in colorectal cancer. Med Oncol. 2012;29(2):919-927.

103. Xi Y, Formentini A, Chien M, et al. Prognostic values of microRNAs in colorectal cancer. Biomark Insights. 2006;2:113-121.

104. Meng X, Wu J, Pan C, et al. Genetic and epigenetic down-regulation of microRNA-212 promotes colorectal tumor metastasis via dysregulation of MnSOD. Gastroenterology. 2013;145(2):426-436. e1.

105. Karaayvaz M, Pal T, Song B, et al. Prognostic significance of miR-215 in colon cancer. Clin Colorectal Cancer. 2011;10(4):340-347.

106. Christensen LL, Tobiasen H, Holm A, et al; COLOFOL steering group. MiRNA-362-3p induces cell cycle arrest through targeting of E2F1, USF2 and PTPN1 and is associated with recurrence of colorectal cancer. Int J Cancer. 2013;133(1):67-78.

107. Yamashita S, Yamamoto H, Mimori K, et al. MicroRNA-372 is associated with poor prognosis in colorectal cancer. Oncology. 2012;82(4):205-212.

108. Yang IP, Tsai HL, Huang CW, et al. The functional significance of microRNA-29c in patients with colorectal cancer: a potential circulating biomarker for predicting early relapse. PLoS One. 2013; 8(6):e66842.

109. Cheng H, Zhang L, Cogdell DE, et al. Circulating plasma MiR-141 is a novel biomarker for metastatic colon cancer and predicts poor prognosis. PLoS One. 2011;6(3):e17745.

110. Pu XX, Huang GL, Guo HQ, et al. Circulating miR-221 directly amplified from plasma is a potential diagnostic and prognostic marker of colorectal cancer and is correlated with p53 expression. J Gastroenterol Hepatol. 2010;25(10):1674-1680. 


\section{Publish your work in this journal}

Cancer Management and Research is an international, peer-reviewed open access journal focusing on cancer research and the optimal use of preventative and integrated treatment interventions to achieve improved outcomes, enhanced survival and quality of life for the cancer patient. The journal welcomes original research, clinical \& epidemiological

studies, reviews \& evaluations, guidelines, expert opinion \& commentary, case reports \& extended reports. The manuscript management system is completely online and includes a very quick and fair peerreview system, which is all easy to use. Visit http://www.dovepress.com/ testimonials.php to read real quotes from published authors.

Submit your manuscript here: http://www.dovepress.com/cancer-management-and-research-journal 